\title{
Sampling zeros and the Euler-Frobenius polynomials
}

\author{
Steven R. Weller \\ Dept. of Electrical and Computer \\ Engineering, \\ University of Newcastle, Australia. \\ Email:steve@ee.newcastle.edu.au \\ Brett Ninness \\ Dept. of Electrical and Computer \\ Engineering, \\ University of Newcastle, Australia. \\ Email: brett@ee.newcastle.edu.au
}

\begin{abstract}
In this paper, we show that the zeros of sampled-data systems resulting from rapid sampling of continuoustime systems preceded by a zero-order hold $(\mathrm{ZOH})$ are the roots of the Euler-Frobenius polynomials, the properties of which have been studied in the context of cardinal spline interpolation and, more recently, wavelets. Using known properties of the Euler-Frobenius polynomials, we prove two conjectures of Hagiwara and coworkers, the first of which concerns the simplicity, negative realness and interlacing properties of the sampling zeros of $\mathrm{ZOH}$ - and first-order hold (FOH-) sampled systems. To prove the second conjecture, we show that in the fast sampling limit, and as the continuous-time relative degree increases, the largest sampling zero for FOH-sampled systems approaches $1 / e$, where $e$ is the base of the natural logarithm.
\end{abstract}

\section{Introduction}

The zeros of discrete-time systems obtained via zeroorder hold ( $\mathrm{ZOH})$ sampling of continuous-time systems play an important role in the design of digital controllers. For single-input, single-output (SISO) continuous-time systems having relative degree $p$, the corresponding discrete-time system obtained by $\mathrm{ZOH}$ sampling has unity relative degree for all but a finite set of sampling periods. The additional $p-1$ discretetime zeros are an artefact of the sampling process, and are called the sampling zeros.

When a continuous-time system is $\mathrm{ZOH}$-sampled with sampling period $T$, it is well known that the

\author{
W. Moran \\ School of Information Science and \\ Technology, \\ Flinders University, Australia. \\ Email:bill@maths .flinders .edu.au
}

\author{
A.D. Pollington \\ Dept. of Mathematics, \\ Brigham Young University, Provo, UT \\ 84602 , U.S.A. \\ Email: andy@math.byu.edu
}

continuous-time poles $\lambda_{i}$ are transformed as [1]

$$
\lambda_{i} \rightarrow e^{\lambda_{i} T} .
$$

For zeros, the situation is considerably more complicated, and no simple transformation is known which shows how continuous-time zeros are mapped to the zeros of the corresponding discrete-time model under ZOH-sampling. In the fast and slow sampling limits (namely, as $T \rightarrow 0$ and $T \rightarrow \infty$, respectively), it is possible to make more precise statements about the location of the corresponding discrete-time zeros; see, for example $[2,3,4,5]$. In particular, for continuous-time systems having relative degree $p$ and $\mathrm{ZOH}$-sampled with sampling period $T \rightarrow 0$, it is known that all finite continuous-time zeros are mapped to the point $z=1$, while the remaining $p-1$ zeros are mapped to the roots of a symmetric polynomial whose integer-valued coefficients depend only on $p[2,3]$. It is these latter zeros - the so-called limiting sampling zeros-which we consider in this paper.

Recently, Hagiwara et al. [4] have shown that similar conclusions conerning the limiting sampling zeros are possible if the zero-order hold is replaced by a first-order hold (FOH); see Theorem 2.2 for a precise statement. In particular, the sampling zeros of $\mathrm{FOH}-$ sampled systems in the fast sampling limit can be obtained as the roots of integer-valued polynomials whose coefficients depend orly on the continuous-time relative degree. Moreover, the polynomials concerned can be readily obtained from two of the $\mathrm{ZOH}$-sampling zeros of successive degrees. Based on strong numerical evidence (see the table on page 1334 of [4]), Hagiwara and co-workers made a three part conjecture concerning the properties of the limiting zeros arising from $\mathrm{ZOH}$ - and FOH-sampling. The first two parts of the conjecture 
concern the simplicity and negative realness (realness) of the roots of $\mathrm{ZOH}$-sampled (FOH-sampled) systems, and the interlacing properties of these roots. In the third part of the conjecture, the authors of [4] made the intriguing observation that, as the continuous-time relative degree increases, the largest root of the $\mathrm{FOH}-$ sampling polynomial appears to converge to $1 / e$, where $e$ is the base of the natural logarithm.

Hagiwara et al. [4] showed that the second part of the conjecture followed from the first, but left unresolved the first and third parts. In this paper, we establish the remaining two parts of the Hagiwara conjecture.

This paper is organized as follows. In Section 2, we review the notion of sampling zeros for both $\mathrm{ZOH}$ - and FOH-sampled systems, and recall a two part conjecture of Hagiwara, Yuasa and Araki [4], the first part of which concerns the simplicity, negative realness, and interlacing properties of sampling zeros of $\mathrm{ZOH}$-sampled systems. We also establish a differential recurrence relation satisfied by the polynomials shown by Aström, Hagander and Sternby [2] to have as roots the sampling zeros of ZOH-sampled systems. In Section 3, we show that the polynomials appearing in [2] are in fact the Euler-Frobenius polynomials, the properties of which have been studied in the context of cardinal spline interpolation $[6,7]$ and, more recently, wavelets [8]. The simplicity and negative realness of the sampling zeros then follows from known properties of the Euler-Frobenius polynomials, while the conjectured interlacing of sampling zeros associated with continuoustime systems of progressively higher relative degrees can be established using the differential recurrence relation satisfied by the polynomials. In Section 4, we prove the second component of the conjecture of Hagiwara and co-workers, namely that as the continuous-time relative degree increases without bound, the largest (i.e. most positive) sampling zero of FOH-sampled systems tends to $1 / e$, where $e$ is the base of the natural logarithm.

\section{Sampling zeros}

It is well known that when the input of a continuoustime dynamical system described by a rational transfer functions $G(s)$ is generated by the piecewise constant output of a zero-order hold ( $\mathrm{ZOH})$, the system output at instants of time (appropriately synchronized with the $\mathrm{ZOH}$ ) can be found using the $z$-transform [1]. In particular, the discrete-time transfer function (or pulsetransfer function) providing the link between input and output samples with sampling period $T$ is given by

$$
G_{0}(z)=Z\left[\frac{1-e^{-s T}}{s} G(s)\right]
$$

where $Z[\cdot]$ denotes the $z$-transform. Likewise, when the system input is generated by a first-order causal extrapolation of sampled values, the sampled inputs and outputs are linked via the first-order hold ( $\mathrm{FOH})-$ equivalent transfer function [4]

$$
G_{1}(z)=Z\left[\frac{1+T s}{T s^{2}}\left(1-e^{-s T}\right)^{2} G(s)\right] .
$$

While the mapping of poles of $G(s)$ under (2) and (3) is readily established, it is difficult to say much about the mapping of finite zeros other than in the limit of fast $(T \rightarrow 0)$ or slow $(T \rightarrow \infty)$ sampling $[2,3,4]$. The following two Theorems summarize the behaviour of the sampled-data models arising from the $\mathrm{ZOH}-$ and FOH-sampling of $G(s)$ in the fast sampling limit.

Theorem 2.1 (Åström et al. [2]) Suppose that $G(s)$ is a strictly proper rational function

$$
G(s)=K \frac{\left(s-\gamma_{1}\right) \cdots\left(s-\gamma_{m}\right)}{\left(s-\lambda_{1}\right) \cdots\left(s-\lambda_{n}\right)}, \quad n>m,
$$

where $\lambda_{i} \in \mathbf{C}(i=1,2, \ldots, n), \gamma_{i} \in \mathbf{C}(i=$ $1,2, \ldots, m)$, and $K \neq 0$. Then, for almost every sampling period $T$, the discrete-time transfer function $G_{0}(z)$ arising from $Z O H$-sampling of (4) has $n-1 z e-$ ros. Furthermore, $G_{0}(z)$ approaches

$$
K \frac{T^{n-m}}{(n-m) !} \frac{(z-1)^{m} B_{n-m}(z)}{(z-1)^{n}}
$$

as $T \rightarrow 0$, where $B_{n-m}(z)$ is the reciprocal polynomial given by

$$
B_{p}(z)=b_{1}^{p} z^{p-1}+b_{2}^{p} z^{p-2}+\cdots+b_{p}^{p}, \quad p \geq 1,
$$

where

$$
b_{k}^{p}=\sum_{l=1}^{k}(-1)^{k-l} l^{p}\left(\begin{array}{c}
n+1 \\
k-l
\end{array}\right), \quad k=1, \ldots, p .
$$

Theorem 2.2 (Hagiwara et al. [4]) Suppose that $G(s)$ is a strictly proper rational function given by (4). Then, for almost every sampling period $T$, the discrete-time transfer function $G_{1}(z)$ arising from FOH-sampling of (4) has $n-1$ zeros. Furthermore, $G_{1}(z)$ approaches

$$
K \frac{T^{n-m}}{(n-m+1) !} \frac{(z-1)^{m} C_{n-m}(z)}{z(z-1)^{n}}
$$

as $T \rightarrow 0$, where $C_{n-m}(z)$ is given by

$$
C_{p}(z)=B_{p+1}(z)+(p+1)(z-1) B_{p}(z), \quad p \geq 1 .
$$


These Theorems suggest that the $m$ so-called limiting zeros approaching $z=1$ correspond to the mapping of the finite zeros $\gamma_{1}, \gamma_{2}, \ldots, \gamma_{m}$, while the remaining $n-m-1$ (or $n-m$ ) zeros arise via the $\mathrm{ZOH}$ (or FOH) sampling process. Hagiwara et al. [4] have justified this assertion, and the $m$ limiting zeros approaching $z=1$ are therefore referred to as the intrinsic zeros, while the zeros approaching the roots of $B_{p}(z)$ or $C_{p}(z)$, where $p=n-m$ is the continuous-time relative degree, are the limiting sampling zeros, also known as discretization zeros.

By evaluating the roots of the polynomials $B_{p}(z)$ and $C_{p}(z)$ for $p=1,2, \ldots, 50$, Hagiwara and co-workers produced compelling numerical evidence to support the following conjecture:

\section{Conjecture 2.1 (Hagiwara et al. [4])}

(a) All roots of $B_{p}(z)$ are single and negative real for any $p$. Furthermore, the roots of $B_{p}(z)$ interlace the roots of $B_{p+1}(z)$ on the negative real axis.

(b) All roots of $C_{p}(z)$ are single and real for any $p$. Furthermore, the $k$ th smallest root of $C_{p}(z)$ lies between the $k$ th smallest root of $B_{p}(z)$ and the $k$ th smallest root of $B_{p+1}(z)$.

(c) The largest root of $C_{p}(z)$ approaches $z=1 / e(\approx 0.3679)$ as $p \rightarrow \infty$, where $e$ is the base of the natural logarithm.

Hagiwara et al. [4] established that property (a) implies property (b). In sections 3 and 4 of the present paper we establish properties (a) and (c) respectively, thereby completing the proof of the conjecture.

\section{The Euler-Frobenius polynomials}

Using (7) and manipulations with binomial identities, it is a straightforward matter to establish that the coefficients of the limiting zero polynomials $\left\{B_{p}(z)\right\}_{p=1}^{\infty}$ can be computed using the following recursive procedure [2]:

$$
\begin{aligned}
b_{1}^{p}=b_{p}^{p}=1, & \\
b_{k}^{p}=k b_{k}^{p-1}+(p-k+1) b_{k-1}^{p-1}, & k=2, \ldots, p-1 .
\end{aligned}
$$

The following Lemma establishes a differential recurrence relation satisfied by the polynomials $\left\{B_{p}(z)\right\}_{p=1}^{\infty}$ directly, rather than in terms of the individual coefficients, as in (10), (11).

Lemma 3.1 The polynomials $B_{p}(z)$, whose coeffcients are given by $(7)$, satisfy the following differential recurrence relation:

$$
\begin{gathered}
B_{1}(z)=1, \\
B_{p}(z)=(1+(p-1) z) B_{p-1}(z)+z(1-z) B_{p-1}^{\prime}(z), \\
p=2,3, \ldots
\end{gathered}
$$

Proof: The result is true by definition for $p=1$. For $p \geq 2$, the recursion can be verified by direct substitution of (6) into (13), equating coefficients of powers of $z$, and simplification using (11).

In the following definition, we recall the EulerFrobenius polynomials, which arise in the study of cardinal spline interpolation $[9,6,10,8]$.

Definition 3.1 The Euler-Frobenius polynomials $E_{k}(x), k=1,2, \ldots$ are defined by the following Rodriguez formula [9]:

$E_{k}(x)=\frac{(1-x)^{k+2}}{x}\left(x \frac{d}{d x}\right)^{k} \frac{x}{(1-x)^{2}}, \quad E_{0}(x)=1$,

where $\left(x \frac{d}{d x}\right)^{k} f(x)=x \frac{d}{d x}\left(x \frac{d}{d x}\right)^{k-1} f(x)$.

We are now in a position to state the first key result of the paper:

Theorem 3.1 The limiting ZOH sampling zero polynomials are the Euler-Frobenius polynomials.

Proof: The idea of the proof is to show that polynomials satisfying the Rodriguez formula (14) simultaneously satisfy the differential recurrence relation defining the limiting sampling zeros. Due to the inconsistency between the numbering of the initial terms $B_{1}(z)$ and $E_{0}(x)$ (cf. (12) and (14)), we work not with (12) and (13), but rather with the recurrence

$$
\begin{gathered}
B_{0}(z)=1 \\
B_{n}(z)=\begin{array}{l}
(1+n z) B_{n-1}(z)+z(1-z) B_{n-1}^{\prime}(z) \\
n=2,3, \ldots,
\end{array}
\end{gathered}
$$

which leads to the same sequence of polynomials as in [2], but with a numbering consistent with (14).

Following Sobolev [9], we introduce the polynomials $K_{k}(y)$ related to $E_{k}(x)$ as follows:

$$
\begin{aligned}
K_{k}(y) & =(y-1)^{k} E_{k}\left(\frac{y+1}{y-1}\right), \\
E_{k}(x) & =2^{-k}(x-1) K_{k}\left(\frac{x+1}{x-1}\right),
\end{aligned}
$$


where the $K_{k}(y)$ satisfy the recurrence relation [9]

$$
K_{k}(y)=\frac{d}{d y}\left[\left(y^{2}-1\right) K_{k-1}(y)\right] .
$$

From (19), the change of variables $y=(x+1) /(x-1)$ yields

$$
\begin{aligned}
K_{k}\left(\frac{x+1}{x-1}\right)= & -\frac{(x-1)^{2}}{2} \frac{d}{d x}\left(\frac{4 x}{(x-1)^{2}} K_{k-1}\left(\frac{x+1}{x-1}\right)\right) \\
= & -2 x \frac{d}{d x} K_{k-1}\left(\frac{x+1}{x-1}\right)+ \\
& K_{k-1}\left(\frac{x+1}{x-1}\right) 2 \frac{x+1}{x-1},
\end{aligned}
$$

from which it follows that

$$
\begin{aligned}
E_{k}(x)= & 2^{-k}(x-1)^{k} K_{k}\left(\frac{x+1}{x-1}\right) \\
= & -2^{-k+1} x(x-1)^{k} \frac{d}{d x} K_{k-1}\left(\frac{x+1}{x-1}\right)+ \\
& \underbrace{2^{-(k-1)}(x-1)^{k-1} K_{k-1}\left(\frac{x+1}{x-1}\right)(x+1)}_{(x+1) E_{k-1}(x)}
\end{aligned}
$$

From (18),

$$
\begin{aligned}
\frac{d}{d x} E_{k-1}(x)= & 2^{-k+1}\left((x-1)^{k-1} \frac{d}{d x} K_{k-1}\left(\frac{x+1}{x-1}\right)\right. \\
& \left.+K_{k-1}\left(\frac{x+1}{x-1}\right)(k-1)(x-1)^{k-2}\right),
\end{aligned}
$$

so that the first term in (21) is given by

$$
\begin{gathered}
-x(x-1) \underbrace{2^{-k+1}(x-1)^{k-1} \frac{d}{d x} K_{k-1}\left(\frac{x+1}{x-1}\right)}_{E_{k-1}(x)}= \\
-x(x-1)\left(\frac{d}{d x} E_{k-1}(x)-2^{-k+1} K_{k-1}\left(\frac{x+1}{x-1}\right)\right. \\
\left.(k-1)(x-1)^{k-2}\right) .
\end{gathered}
$$

Substituting this expression into (21) gives

$$
\begin{aligned}
E_{k}(x)= & x(1-x)\left(\frac{d}{d x} E_{k-1}(x)\right. \\
& \left.-2^{-k+1} K_{k-1}\left(\frac{x+1}{x-1}\right)(k-1)(x-1)^{k-2}\right) \\
& +(x+1) E_{k-1}(x) \\
= & x(1-x) \frac{d}{d x} E_{k-1}(x) \\
& +x(k-1) \underbrace{2^{-k+1}(x-1)^{k-1} K_{k-1}\left(\frac{x+1}{x-1}\right)}_{E_{k-1}(x)} \\
& +E_{k-1}(x)+x E_{k-1}(x) \\
= & (1+k x) E_{k-1}(x)+x(1-x) \frac{d}{d x} E_{k-1}(x),
\end{aligned}
$$

and the result is proved.
Corollary 3.1 In the fast sampling limit, the sampling zeros arising from the ZOH-sampling of continuoustime systems of relative degree 2 or greater are simple and negative real.

Proof: These are known properties of the EulerFrobenius polynomials; see [9], for example.

Lemma 3.2 In the fast sampling limit, the sampling zeros arising from the $Z O H$-sampling of continuoustime systems having progressively higher relative degrees are interlaced on the negative real axis.

Proof: Consider the recurrence relation (13) evaluated at any of the $(p-2) \operatorname{roots} z_{i}^{*}$ of $B_{p-1}(z)$ :

$$
B_{p}\left(z_{i}^{*}\right)=z_{i}^{*}\left(1-z_{i}^{*}\right) B_{p-1}^{\prime}\left(z_{i}^{*}\right) .
$$

From Corollary 3.1, all roots of $B_{p-1}(z)$ are negative real, so that $z_{i}^{*}\left(1-z_{i}^{*}\right)<0$ and thus the sign of $B_{p}\left(z_{i}^{*}\right)$ is opposite that of $B_{p-1}^{\prime}\left(z_{i}^{*}\right)$. Since $B_{p}(z)=1$ for all $p \geq$ 1 , it follows from the simplicity of the $z_{i}^{*}$ and the Mean Value Theorem that the $i$ th root of $B_{p}(z)$ lies strictly to the right of the corresponding root of $B_{p-1}(z)$ for $i=1,2, \ldots, p-2$. Since

$$
\lim _{z \rightarrow-\infty} B_{p}(z) \begin{array}{cc}
<0, & p \text { even } \\
>0, & p \text { odd }
\end{array}
$$

$\lim _{z \rightarrow-\infty} B_{p}(z)$ and $\lim _{z \rightarrow-\infty} B_{p-1}(z)$ have opposite signs. From the Mean Value Theorem, there must exist a root of $B_{p}(z)$ to the left of the most negative root of $B_{p-1}(z)$, and the proof is completed.

Taken together, Corollary 3.1 and Lemma 3.2 constitute a proof of Conjecture 2.1 (a), and hence from [4], Conjecture 2.1 (b) is also proved.

\section{The largest zero of FOH-sampled systems}

In this section, we prove the third part of the Hagiwara conjecture, namely that in the fast sampling limit, the largest sampling zero of FOH-sampled systems approaches $1 / e$ as the continuous-time relative degree increases. The proof does not rely heavily on the fact that the limiting sampling zeros of $\mathrm{ZOH}$-sampled systems are the roots of the Euler-Frobenius polynomials, but does use a key change of variables and a series expansion introduced by Sobolev in his study of the roots of these polynomials [9].

Theorem 4.1 In the fast sampling limit, the most positive sampling zero arising from the FOH-sampling of continuous-time systems having relative degree $p$ approaches $1 /$ e as $p \rightarrow \infty$. 
Proof: The sequence of polynomials of interest is generated by (9), where (from Theorem 3.1), the polynomials $B_{p}(z)$ satisfy the Rodriguez formula (14). Following Sobolev [9], a key ingredient is to make the substitution $z=e^{\pi \theta}$, leading to

$$
B_{k}\left(e^{\pi \theta}\right)=\left(\frac{2}{\pi}\right)^{k} e^{\pi \theta k / 2} \sinh ^{k+2}\left(\frac{\pi \theta}{2}\right) \frac{d^{k}}{d \theta^{k}} \frac{(\pi / 2)^{2}}{\sinh ^{2} \pi \theta / 2} .
$$

Define

$$
S_{k}(\theta)=\frac{d^{k}}{d \theta^{k}} \frac{(\pi / 2)^{2}}{\sinh ^{2} \pi \theta / 2}
$$

so that the roots of $C_{k}$ other than 0 correspond to solutions of

$$
\bar{S}_{k}(\theta)=0 \text {, }
$$

where

$$
\bar{S}_{k}(\theta)=(k+1) S_{k}(\theta)-\frac{1}{\pi} S_{k+1}(\theta) .
$$

We note that the Cauchy expansion of $1 / \sinh ^{2} \pi \theta$ gives

$$
S_{k}(\theta)=-(k+1) !(-j)^{k} \sum_{n=-\infty}^{\infty} \frac{1}{(j \theta-2 n)^{k+2}} .
$$

The intuition behind the proof is that for $k$ sufficiently large, the central term

$$
h_{k}(\theta)=-(k+1) !(-j)^{k} \frac{1}{(j \theta)^{k+2}}
$$

dominates the infinite sum (26), so that the solutions of (24) are approximately given by the roots of

$$
\begin{aligned}
f_{k}(\theta) & =(k+1) h_{k}(\theta)-\frac{1}{\pi} h_{k+1}(\theta) \\
& =\frac{(-1)^{k}(k+1) !}{\theta^{k+2}}\left[k+1+\frac{1}{\pi} \frac{k+2}{\theta}\right] .
\end{aligned}
$$

For $k$ sufficiently large, the single root of $f_{k}(\theta)$ approaches $-1 / \pi$, and thus since $z=e^{\pi \theta}=1 / e$, we are done.

To make the argument rigourous, we use Rouché's Theorem $[11, \mathrm{p} .300]$ to show that for $k$ sufficiently large, the contribution to $\bar{S}_{k}(\theta)$ from the neglected (non-central) terms

$$
c_{k}(\theta)=S_{k}(\theta)-h_{k}(\theta)
$$

is vanishingly small in the sense that $f_{k}(\theta)$ and $\bar{S}_{k}(\theta)$ have the same number of zeros as $k \rightarrow \infty$. Consider the remainder term

$$
g_{k}(\theta)=(k+1) c_{k}(\theta)-\frac{1}{\pi} c_{k+1}(\theta) .
$$

Using (26) we have

$$
\left|g_{k}(\theta)\right| \leq 4(k+1)(k+1) ! \sum_{n=1}^{\infty} \frac{1}{\left(x^{2}+(2 n-y)^{2}\right)^{(k+2) / 2}} .
$$

Now use an Integral Test estimate for the right hand side to obtain

$$
\begin{aligned}
\left|g_{k}(\theta)\right| \leq & 4(k+2) \frac{1}{\left(x^{2}+(2-y)^{2}\right)^{(k+2) / 2}} \\
& +\int_{1}^{\infty} \frac{1}{\left(x^{2}+(2 u-y)^{2}\right)^{(k+2) / 2}} d u \\
\leq & 4(k+2) \frac{1}{\left(x^{2}+(2-y)^{2}\right)^{(k+2) / 2}} \\
& +\frac{1}{2} \int_{2-y}^{\infty} \frac{1}{\left(x^{2}+u^{2}\right)^{(k+2) / 2}} d u \\
\leq & 4(k+2) \frac{1}{\left(x^{2}+(2-y)^{2}\right)^{(k) / 2}} \\
& \left(1+\int_{2-y}^{\infty} \frac{1}{\left(x^{2}+u^{2}\right)} d u\right) \\
\leq & \frac{C(k+2) !}{\left(x^{2}+(2-\epsilon)^{2}\right)^{k / 2}}
\end{aligned}
$$

for some constant $C$. Here we have $\theta=x+j \xi$ where $|\xi|=y \leq \epsilon<2$.

Consider the value of $f_{k}$ on a contour $\Omega$ defined as the boundary of a square, centred on the point $-1 / \pi$, having sides of length $2 \epsilon$, and taken in the counterclockwise direction:

$$
\begin{aligned}
\left|f_{k}(\theta)\right| & =\frac{((k+1) !}{|\theta|^{k+2}}\left|k+1+\frac{1}{\pi} \frac{k+2}{\theta}\right| \\
& \geq \frac{(k+2) !}{\left(x^{2}+y^{2}\right)^{(k+2) / 2}}\left(\left(\epsilon^{2}+x^{2}\right)^{1 / 2}-\frac{1}{k+2}\right) .
\end{aligned}
$$

Thus for any given $\epsilon$ we can find $K$ such that if $k \geq K$,

$$
\left|f_{k}(\theta)\right|>\left|g_{k}(\theta)\right|
$$

for $\theta$ on contour $\Omega$. By Rouché's Theorem, $f_{k}(\theta)$ and $f_{k}(\theta)+g_{k}(\theta)=\bar{S}_{k}(\theta)$ have the same number of zeros inside $\Omega$. Since $f_{k}$ has exactly one zero for $k$ sufficiently large, so does equation (24), and by taking $\epsilon$ small we can show that for large $k$ the zero is close to $-1 / \pi$. Thus as $k \rightarrow \infty$, there are no other real roots of $C_{k}(z)$ larger than $1 / e$, and the result is proved.

\section{Conclusions}

In this paper, we have established that the sequence of polynomials whose roots are the limiting sampling zeros of ZOH-sampled systems are in fact the EulerFrobenius polynomials. Several conjectured properties of the limiting sampling zeros of $\mathrm{ZOH}-$ and FOHsampled systems then follow immediately, or can be established from a differential recurrence formula satisfied by the Euler-Frobenius polynomials. Finally, a conjecture by Hagiwara and co-workers that the largest limiting sampling zero of FOH-sampled systems approaches $1 / e$ as the continuous-time relative degree increases has been proved. Since ZOH-equivalent models are approximations of a particular form, it is not 
entirely surprising that a sequence of polynomials occurring in the study of interpolation problems should arise in the analysis of sampled-data systems. Further work is needed, however, to clarify the nature of the connection.

\section{References}

[1] K.J. Åström and B. Wittenmark, Computer Controlled Systems: Theory and Design, Prentice-Hall, Englewood Cliffs, NJ, 1984.

[2] K.J. Åström, P. Hagander, and J. Sternby, "Zeros of sampled systems", Automatica, vol. 20, no. 1, pp. 21-38, 1984.

[3] V.A. Bondarko, "Discretization of continuous linear dynamic systems. Analysis of the methods", Systems $\mathcal{B}$ Control Letters, vol. 5, pp. 97-101, November 1984.

[4] T. Hagiwara, T. Yuasa, and M. Araki, "Stability of the limiting zeros of sampled-data systems with zeroand first-order holds", Int. J. Control, vol. 58, no. 6, pp. 1325-1346, 1993.

[5] B. Mårtensson, "Zeros of sampled systems", Master's thesis, Dept. of Automatic Control, Lund, Sweden, 1982, TFRT-5266.

[6] F. Dubeau and J. Savoie, "On the roots of orthogonal polynomials and Euler-Frobenius polynomials", J. Math. Anal. Appl., vol. 196, no. 1, pp. 84-98, November 151995.

[7] M. Reimer, "The main roots of the EulerFrobenius polynomials", J. Approx. Theory, vol. 45, no. 4, pp. 358-362, December 1985.

[8] C.K. Chui, An Introduction to Wavelets, Academic Press, New York, 1992.

[9] S.L. Sobolev, "On the roots of Euler polynomials", Soviet Math. Dokl., vol. 18, no. 4, pp. 935-938, 1977.

[10] F. Dubeau and J. Savoie, "Explicit error bounds for spline interpolation on a uniform partition", $J$. Approx. Theory, vol. 82, pp. 1-14, 1995.

[11] R.V. Churchill, J.W. Brown, and R.F. Verhey, Complex Variables and Applications, McGraw-Hill, third edition, 1974. 